\title{
Research of control network configuration technology based on function block
}

\author{
Jie Ren ${ }^{1, \mathrm{a}}$ Zongzhi Zhu ${ }^{2, \mathrm{~b}}$ Cuiping $\mathrm{Pu}{ }^{1, \mathrm{c}} \mathrm{An} \mathrm{Yu}{ }^{1, \mathrm{~d}}$ \\ ${ }^{1}$ Kunming University, Kunming, Yunnan, 650214,China \\ ${ }^{2}$ Zhejiang Supcon Research CO LTD, Hangzhou , 310053, China \\ arenjie-9999@163.com, bzhu_zz1106@163.com, 'pucuiping@126.com, ${ }^{d}$ 34018958@qq.com
}

Keywords: EPA; function block;configuration

\begin{abstract}
The function block model and configuration technology were studied.A method of function block instantiation is advanced in allusion to the problem that the number of function block is fixed.At the same time, the parallelism was employed for the schedule of function blocks. And the advantages in the complex control system were analyzed. The study has practical significance to engineering application.
\end{abstract}

\section{Introduction}

Based on the establishment of the EPA standard of industrial automation control system is distributed control system, all the control functions is through the distribution in the mutual operation between field devices on the various functional blocks to complete, so the EPA device configuration is actually blocks for all kinds of functions of equipment configuration and scheduling [1]. EPA function block is the intelligent control module for industry field, and it is the basic element of EPA field control system. Functional block model provides a general structured method. the various functions required for the control system are divided into completely independent and complete different control function modules, and the public features are labeled[2].

Function block cancel the field control station of the traditional DCS control system . The control is completely decentralized to the field equipment, through the field instrument can constitute the control loop.the complete decentralized control is realized.the reliability, self-discipline and flexibility of the system are improved[1].

The type and number of function blocks in EPA Ethernet equipment are mostly fixed.The manufacturers can not predict a device applications, therefore cannot ante control requirements placed specific types and quantities of function block. Also the resources of embedded system are limited, the type and number of the function blocks in devices are limited. when the particular control task is completed,the equipment containing the desired functional blocks must be selected, and the appropriate logic configuration and physical installation are necessary. This adds to the complexity of the use, limits the flexibility of the use, and caused waste to a certain extent. The solution of the function block instantiation based on configuration software is put forward, which improves the flexibility of the function block, and simplifies the use of the function block and saves the cost.

\section{EPA Function Block Specification}

Field equipment model.The field equipment model is shown in Figure 1, including EPA communication interface, EPA function blocks and process interface, and it also includes the sensor, actuator and other equipments and objects. EPA function blocks are applied on user layerof EPA protocol model.EPA user layer provides function block application of control strategy for users, and for function blocks provides support for matters related to the protocol.the field device can realize the control function of control system and the resource management. The EPA user layer provides the interface of the production process for the user [2]. The field equipment can be connected to the 
network and other equipment for communication,through the communication interface.The process interface provides a mapping between physical processes and resources.Information that is exchanged with physical processes is presented to the resource in the form of data or time or both.

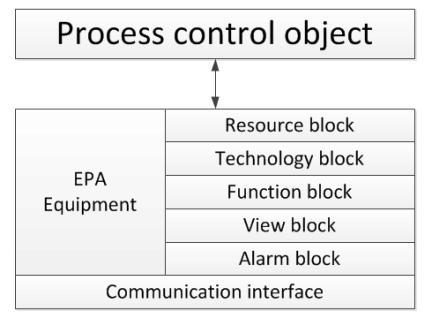

Fig. 1 Field Equipment Model
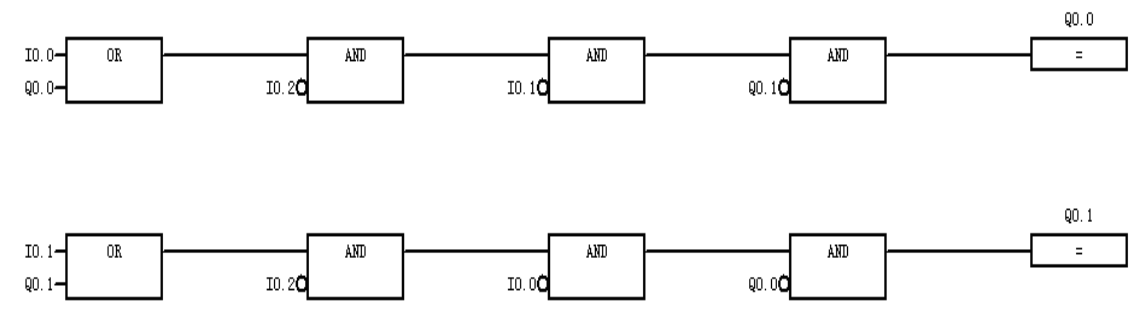

Fig. 2 Logic Control Loop

Function block specification. The function blocks are the basic atomic elements that can not be divided in EPA system. there is aentity contain 'ning the executable algorithm, with input and output parameters and the internal parameters and external interface for input and output time or data. The control function of the system is accomplished by the event transfer or data exchange cooperative with the function block of the same field equipment or different field equipment[3].

The fieldbus control system are composed of the digital field equipments and the monitoring equipments. All kinds of equipments are connected together by the communication network, which provide the $\mathrm{I} / \mathrm{O}$ and control function for the automation process and operation.Control strategy is implemented by the functional block application process [3]. the functional block application process are used to implement the functions required by users. They are the basic part of the EPA fieldbus control system.. They provide a complete operating environment for the realization of the control system function, and support the information exchange between different functional blocks [4].

This will ensure that the function block instanceones of one or more EPA devices can order in accordance with the provisions of the configuration implementation through the function block scheduling. so as to the function blocks are mutually coordinated operation, complete control function [5].

\section{Configuration Technology Based on the Function Blocks}

According to the EPA distributed control system application scheme, the configuration link input and output parameters of function blocks instance in the EPA network in a certain way. The control link is formed,and the parameters and periodic packets of the network are configured in the EPA function block instance. According to the function blocks application process configuration, theconfiguration program specify a EPA link object for each communication link, according to the relationship of the communication link between the function blocks instance.It used to specify the communication access path of each communication relationship.

Equipment configuration.After the EPA device is powered up, it automatically sends the message of the device to declare the service and tells the existence of deviceexistence in the EPA network .After the configuration software receives the device declaration, it analysises the DeviceID field in the EPA message.This field is a unique identifier for each device in the EPA network. Then it read the management information base of equipment .

The equipment management module is the core module in the EPA configuration. The equipment classes, function blocks classes, parameters base classes, and member base classes form the class of device management. The main information of the device class is the function block information, and a device object contains one or more functional blocks. The function block classes is a parameter information, and a structure parameter object contains one or more member objects. There are four parameter classes, one is the base class, the other three parameter classes are derived from the base class, the member class have three species, one is the member base class, the other two member classes are derived from the member base class. 
Control link configuration.The EPA link object description link relations or the access path between a function block instance output parameters with another function block instance input parameters, and specify the communication role of a device in the communication relationship.The each EPA link object is uniquely identified in the device by the linked object identifier ObjectID. In the EPA system, the communication link between the input / output parameters of the functional block instance is composed of three levels Identification. They are original equipment IP address SourceIPAddress, SourceAppID, SourceObjectID, DestinationIPAddress, DestinationAppID, DestinationObjectID.

Function block scheduling configuration.The left side of the function block graphic are input parameters, right as output parameters. The function blocks can freely mobile location. The function blocks can be connected by lines represent the link relation between them.Each connection line represents the linked object in an EPA. The link relationship between function blocks form control loop. The function blocks configuration module realizes the analog function blocks on the interface and the function blocks in equipment in different classes,also between the two with a pointer to the corresponding.The forms of the function blocks on the interface are the function blocks, the port and the wire.they design the corresponding data structure for them,in order to correctly describe the function block of the device and file.

\section{Function Blocks Instance}

The function blocks can be distributed in the same equipment, and can be dispersed in many devices.The appropriate function blocks configuration complete control algorithm. The function blocks Instance is the use of the configuration software to dynamically add and remove the function blocks in the field equipment, so that the resources of the equipment can be reasonably utilized.Dynamic addition and removal the types of functional blocks can make the standard function blocks, and the flexible function blocks that the device manufacturer defined. The addition and removal of the function blocks can be performed in any of the devices, which not only breaks through function block location restriction of the physical device installation position, so that the equipment application is more flexible, and we more fully reasonable used the resources of all equipment in the field.

Each instantiation of function block has the ID of the fixed equipment and identifies the resources of fixed equipment [7]. The functional blocks associated with the device hardware are defined as the static function blocks, and the function blocks unrelated to the device's hardware are defined as the dynamic function blocks. Static function block can only be instantiated once, each static function block instance number corresponding to the corresponding hardware interface input and output channels.If the function blocks associated with the device hardware are dynamically instanced, the ID number allocated beyond the hardware range will not be able to obtain the input output data. The instantiation of dynamic function blocks are to traverse the function block node in XML file through DOM document object, and find the starting function block of the corresponding type.In the corresponding type of function block nodes, it traverses function blocks ID, it allocates the new function block ID.

In practical engineering, we often need to use complex logic control, need to use multiple logical blocks. The dynamic instance of the functional blocks can avoid the problem of insufficient number of functional blocks. and a specific example is the realization of the function block.A specific example verifies the realization of the function block instantiation.Figure 2 shows a logical control loop .that accomplished the reversible control of the motor with double locks. The control loop is compiled and downloaded to the EPA device. Three DI function blocks are used, two DO function blocks are used, 8 functional blocks are dynamically instanced.The test results prove that the control loop is correct and the method of function blocks instance is correct.

A device can have one or a plurality of functional blocks of the different kinds .However, at a certain time, a device there can be at most a function block scheduling [8], due to the limitations of CPU.The functional blocks in a same device are a serial execution. For some complex control process, 
we need to multiple function blocks collaborative work. The function blocks are distributed to a plurality of devices for scheduling, so that they are executed in parallel, In order to ensure the completion of the preset control strategy in a period of internal control,and meet the control requirements and real-time requirements. As shown in Figure 3, FB1 and FB2 of the equipment 3 have a large amount of computation and long execution time.FB2, FB1 were respectively treated with the sampling results of equipment 1 and equipment 2, after FB3 further calculated output. The most of the time CPU of equipment 1,2 is idle, and the CPU resource of device 3 is very tight, it is difficult to guarantee real-time control algorithm. The use of functional block Instance technology reconstruct the parallel control system as shown in Figure 4.It can solve the above problem. FB1 and FB2 are respectively placed in the equipment 1 and equipment 2 .They handle the sampling datas in each of the respective devices and send the results to the device 3 . The response time of the control loop of the parallel execution is largely shortened to a large extent,and the control performance of the system is improved.

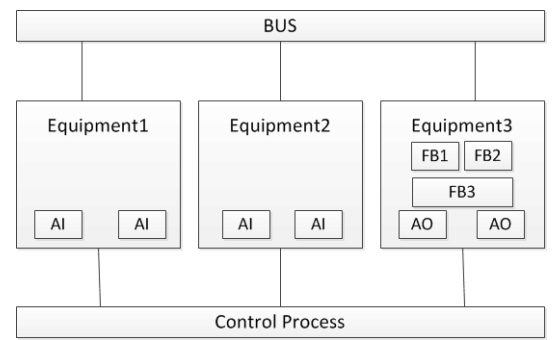

Fig. 3 Serial Execution of Complex Control Function Blocks

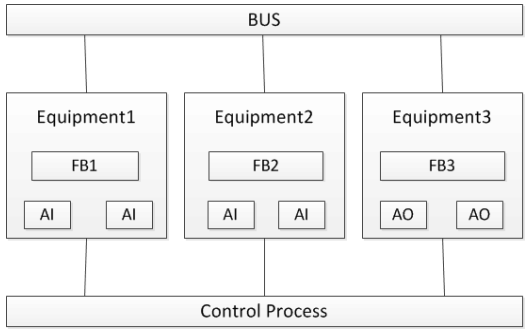

Fig. 4 Parallel Execution of Complex Control Function Blocks

\section{Conclusion}

The function blocks Instance is the use of the configuration software to dynamically add and remove the function blocks in the field equipment, so that the resources of the equipment can be reasonably utilized.For some complex control process, we need to multiple function blocks collaborative work. The function blocks are distributed to a plurality of devices for scheduling, so that they are executed in parallel, In order to ensure the completion of the preset control strategy in a period of internal control,and meet the control requirements and real-time requirements.

\section{Acknowledgements}

This work was financially supported by Applied basic research project of Yunnan Province (2013FZ098) and The scientific research fund project of Education Department of Yunnan province (2013Y257) . Data were supplied by Kunming University Library .In addition, I am grateful to the team members for their patience and good counsel. The anonymous reviewers have also contributed considerably to the publication of this paper.

\section{References}

[1] Ping Wang, Haofei Xie, Qiong Xiao. Industrial Ethernet technology[M]. Science Press2007, p160-177,263-307 
[2] Tong Zhou, Kaidong Xu, Hong Wang.Research on EPA Functional and User Layer Technology.Process Automation Instrumentrumentation[J].Vol.27 No.9 September 2006.p16-18

[3] Yingli Fu, Ping Wang.Design and implementation of function block based on EPA standard.Journal of Chongqiong University of Posts and Telecommunications(Natural Science)[J]. .Vol.18 No.2 Apr 2006.p243-246

[4] IEC 61804 (PAS ） Function Blocks for Process Control [S].Beijing:[S.n.]2002 0409

[5] IEC 61499 (PAS) Function Blocks for Industrial Process Measurement and Control System [S].Beijing:[S.n.]2004 072020051222

[6] Yong Lu, Tianran Wang, Haibin Yu, Hong Wang.Instantiation of Fieldbus Function Block.Information and Control[J]. Vol.33 No.3 June.200.p332-336.

[7] Huili Wang. Funetion Bloek Programming Researchand Configuration Software Design[D]. Dalian:Dalian University of Teehno]og, 2008: p29-38

[8] Songhui Zhang. The Study of Funetion Block on IPMCS Based on IEC61499 Standard [D]. Hangzhou,: ZhejiangUniversity, 2005: p68-69

Author brief introduction: Jie Ren(1981-), Male, Kunming City Yunnan province, Bachelor degree, Lecturer, Research Direction for Control Engineering, renjie-9999@163.com. 